\title{
Experimental study of atomic $4 d$ giant resonances by photoabsorption and photoelectron spectroscopy: Sm, Eu, and Gd
}

\author{
M. Richter, M. Meyer, M. Pahler, T. Prescher, ${ }^{*}$ E. v. Raven, \\ B. Sonntag, and H.-E. Wetzel ${ }^{\dagger}$ \\ II. Institut für Experimentalphysik, Universität Hamburg, Luruper Chaussee 149, \\ D-2000 Hamburg 50, Federal Republic of Germany
}

(Received 26 May 1989)

\begin{abstract}
Excitation and decay of the $4 d^{-1}(4, \epsilon) f$ giant resonances of atomic Sm, Eu, and Gd have been investigated by photoabsorption and photoelectron spectroscopy. The experimental total and partial cross sections are compared to those predicted by many-electron theories. Autoionization into the $4 f^{-1} \epsilon l$ continuum dominates. The character of the giant resonances is closer to that of a $4 d^{-1} 4 f$ resonance than to that of a $4 d^{-1} \epsilon f$ shape resonance encountered for $\mathrm{Ba}$ and $\mathrm{La}$.
\end{abstract}

\section{INTRODUCTION}

The key features of the rare-earth atoms are due to the corelike character of the partly filled $4 f$ shell. Even for the metals the descriptions of the electronic states are normally based on atomic $4 f$ orbitals spatially localized around the nucleus. The strong overlap of the $4 f$ and $4 d$ orbitals has made the $4 d^{-1}(4, \epsilon) f$ giant resonances an important proving ground for many-electron processes. These resonances are excited by the promotion of a $4 d$ electron into a final state, the character of which varies between a $\epsilon f$ continuum state and a bound $4 f$ state $[4 d \rightarrow(4, \epsilon) f$ excitations]. The experimental and theoretical endeavors have been briefly outlined in our previous paper on $\mathrm{Ba}, \mathrm{La}$, and $\mathrm{Ce} .^{1}$ For details the reader is referred to the references quoted therein, especially to the compilation of recent review articles on giant resonances which has been edited by Connerade, Esteva, and Karnatak. ${ }^{2}$ In the investigations on $\mathrm{Ba}, \mathrm{La}$, and $\mathrm{Ce}$ our interest was focused on the "collapse" of the $4 f$ orbital. In the present paper we are mainly dealing with the elements $\mathrm{Sm}, \mathrm{Eu}$, and $\mathrm{Gd}$ which occupy the center of the rareearth series. The corelike $4 f$ shell is half-filled for Eu and Gd. Sm is just short of one $4 f$ electron to complete the $4 f^{7}$ configuration. The $4 f^{7}\left({ }^{8} S_{7 / 2}\right)$ configuration is so stable that it is conserved in Eu and Gd metal, whereas, except for $\mathrm{Ce}, \mathrm{Yb}$, and $\mathrm{Lu}$ the other rare-earth atoms undergo a valence change upon solidification, whereby one $4 f$ electron is promoted to the $5 d$ band. ${ }^{3-6}$ This also holds for bulk Sm but not for the Sm surface atoms, which preserve the $4 f^{6}$ configuration of the free Sm atoms. $^{7}$ The exceptional stability of the $\mathrm{Eu}$ and $\mathrm{Gd}$ $4 f^{7}\left({ }^{8} S_{7 / 2}\right)$ states results in a dramatic simplification of the spectra, because the coupling of the $4 f$ electrons is very unlikely to be changed upon excitation. In many cases the $4 f$ electrons can be treated as spectators. Therefore the $4 d \rightarrow(4, \epsilon) f$ oscillator strength is concentrated in the transitions to a few excited states with $4 f^{7}\left({ }^{8} S_{7 / 2}\right)$ parentage. The reduced complexity of the spectra singles out $\mathrm{Eu}$ and $\mathrm{Gd}$ for thorough tests of theoretical approaches. Total and partial cross sections have been obtained in the relativistic time-dependent local-density approximation (RTDLDA) for all three elements $\mathrm{Sm}, \mathrm{Eu}$, and $\mathrm{Gd} .^{8,9} \mathrm{Eu}$ also successfully has been tackled by the random-phase approximation with exchange (RPAE) ${ }^{10}$ and the many-body perturbation theory (MBPT). ${ }^{11}$

Figure 1 displays the $4 d$ absorption spectra of the elements $\mathrm{Ba}, \mathrm{La}, \mathrm{Ce}, \mathrm{Pr}, \mathrm{Nd}, \mathrm{Sm}, \mathrm{Eu}, \mathrm{Gd}$, and Tb. ${ }^{12-16}$ The experimental procedure has been described in Ref. 1 . The absorption spectra were recorded photoelectrically, thus avoiding the difficulties encountered in determining reliable relative absorption cross sections from photographically registered spectra. Since the density of atoms in the absorbing vapor column has not been determined, only relative cross sections have been obtained. The spectra given in Fig. 1 clearly show the simplification brought about by the stability of the $4 f^{7}\left({ }^{8} S_{7 / 2}\right)$ state. The Eu and Gd spectra display only one strong asymmetric absorption band preceded by a series of weak lines, whereas the spectra of the neighboring elements are characterized by a very broad and structured maximum. These spectra reflect the complex multiplet structure of the excited $4 d^{9}(4, \epsilon) f^{n}$ states populated via $4 d^{10} 4 f^{n-1} \rightarrow 4 d^{9}(4, \epsilon) f^{n}$ excitations and their coupling to the $5 s^{-1}, 5 p^{-1}, 4 f^{-1}$, and $4 d^{-1}$ ionization continua. To reach a full understanding is a very demanding task. For the present state of our understanding the reader is referred to Refs. 1 and 2 and the articles quoted therein. Photoelectron spectroscopy can considerably contribute to the characterization of the $4 d^{9}(4, \epsilon) f^{n}$ states and the excitation and decay mechanism. In the following sections we will concentrate on the spectra of atomic Sm, $\mathrm{Eu}$, and $\mathrm{Gd}$. The photoelectron spectra of the free atoms have been obtained by the same experimental procedure and the same experimental setups as described for $\mathrm{Ba}, \mathrm{La}$, and Ce. ${ }^{1}$ In the last section of the paper we will outline and discuss the $Z$ dependence of the giant resonances. In this context we also will come back to the absorption spectra given in Fig. 1. 


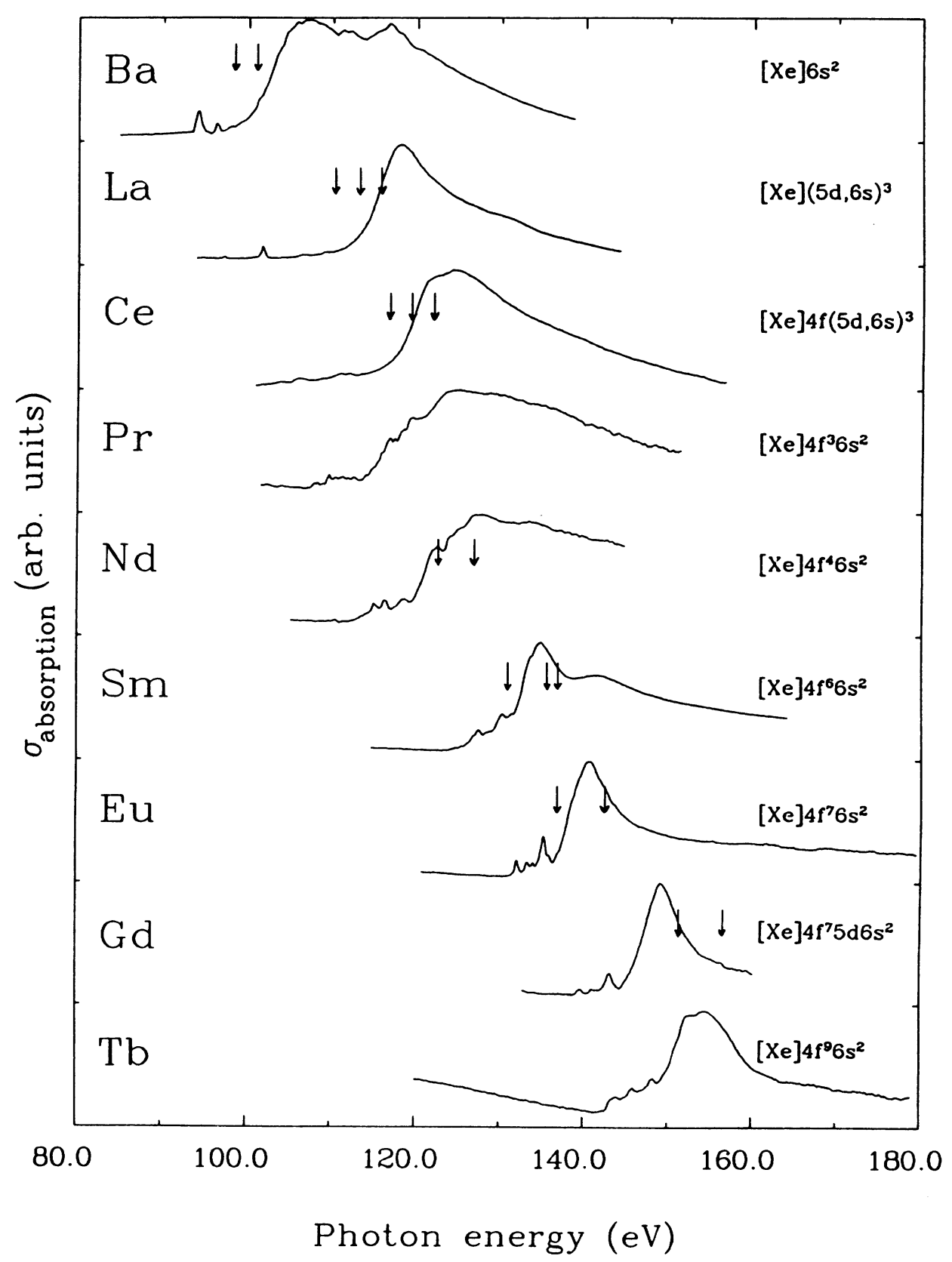

FIG. 1. Photoelectrically detected photoabsorption spectra of $\mathrm{Ba}, \mathrm{La}$, and lanthanides (Refs. 12-16). The arrows mark the position of the $4 d^{-1}$ ionization limits determined from the main photoelectron lines (see Ref. 1 and Tables I and II).

\section{RESULTS AND DISCUSSION}

\section{A. Photoelectron spectra of atomic Sm, Eu, and Gd}

Photoelectron spectra taken at photon energies below, at, and above the $4 d$ giant resonances of atomic $\mathrm{Sm}, \mathrm{Eu}$, and $\mathrm{Gd}$ are shown in Fig. 2. At the evaporation temperature of $\cong 800 \mathrm{~K}$ only the ground-state $\mathrm{Eu}$ [Xe] $4 f^{7} 6 s^{2}\left({ }^{8} S_{7 / 2}\right)$ is populated; therefore all photoelectron lines can be ascribed to excitations out of the ground state. The $6 s^{-1}, 4 f^{-1}, 5 p^{-1}$, and $5 s^{-1}$ photoelectron lines are clearly discernible in the Eu photoelectron spectrum taken at $h v=135.2 \mathrm{eV}$, i.e., below the $4 d$ resonance (center part of Fig. 2). The strong $4 f^{-1}$ line is accompanied by satellite lines due to a recoupling of the $4 f$ electrons or to a shakeup of a $6 s$ valence electron. The splitting of the $5 p^{-1}$ lines is caused by the $5 p$ spin-orbit interaction and the coupling of the open $5 p$ and $4 f$ shells. Satellite emission contributes to the high-energy part of the $5 p^{-1}$ emission. The interaction of the partly filled $5 s$ and $4 f$ shells splits the $5 s^{-1}$ emission into two lines. Resonant $\mathrm{N}_{45} \mathrm{O}_{23} \mathrm{O}_{23}$ Auger decay probably is responsible for 


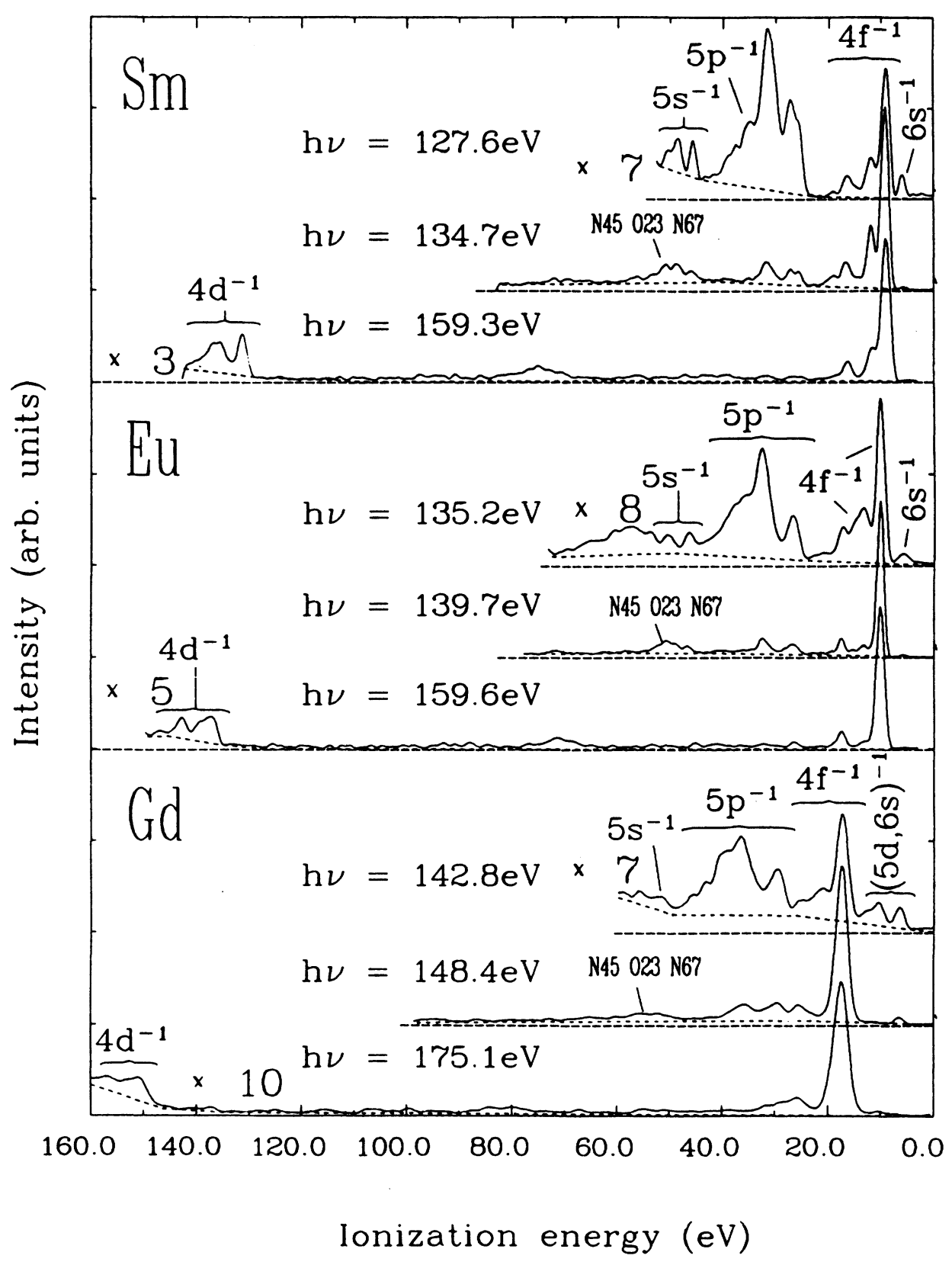

FIG. 2. Photoelectron spectra of atomic Sm, $\mathrm{Eu}$, and $\mathrm{Gd}$ taken at photon energies in the range of the $4 d$ giant resonances.

the intensity above $E_{B}=55 \mathrm{eV}$ in the spectrum taken at $h v=135.2 \mathrm{eV}$.

The $4 d^{-1}$ photoelectron lines can be well observed in the spectrum taken at $h v=159.6 \mathrm{eV}$, i.e., above the giant resonance. Calculations ${ }^{11}$ place the $4 d^{9} 5 s^{2} 5 p^{6} 4 f^{7} 6 s^{2}\left({ }^{9} D,{ }^{7} D\right)$ ionization limits at 139.6 and $159.8 \mathrm{eV}$. In agreement with these calculations we ascribe the $4 d^{-1}$ lines detected in our spectra to the $4 d^{9} 5 s^{2} 5 p^{6} 4 f^{7} 6 s^{2}\left({ }^{9} D\right)$ ionic state split by the $4 d$ spin-orbit interaction. For photon energies above $138 \mathrm{eV}$ the $\mathrm{N}_{45} \mathrm{O}_{23} \mathrm{~N}_{67}$ Auger decay manifests itself in the electron spectra. Our tentative assignment of the photoelectron lines is summarized in Table I. The assignment is based on the tabulated energy values, ${ }^{17}$ the multiconfiguration Hartree-Fock calculations of Pan et al. ${ }^{11}$ and the interpretation of analogous data. 1, 18,19

We started the discussion with $\mathrm{Eu}$ because there are more data available for Eu than for Gd and Sm and the semi-closed-shell character of Eu simplifies the interpretation. We will now turn to the electron spectra of Gd displayed in the bottom part of Fig. 2. At $\cong 1500 \mathrm{~K}$, the temperature required to generate an atomic $\mathbf{G d}$ beam of sufficient density, several Gd $[\mathrm{Xe}] 4 f^{7} 5 d 6 s^{2}\left({ }^{9} D_{J}\right)$ states are populated besides the $\mathrm{Gd}[\mathrm{Xe}] 4 f^{7} 5 d 6 s^{2}\left({ }^{9} D_{2}\right)$ ground 
TABLE I. Ionization energies of atomic $\mathrm{Eu}[\mathrm{Kr}] 4 d^{10} 5 s^{2} 5 p^{6} 4 f^{7} 6 s^{2}\left({ }^{8} S_{7 / 2}\right)$ and $\mathrm{Gd}$ $[\mathrm{Kr}] 4 d^{10} 5 s^{2} 5 p^{6} 4 f^{7} 5 d 6 s^{2}\left({ }^{9} D_{2, \ldots, 6}\right)$. The $(5 d, 6 s)^{-1}$ energies from optical data (Ref. 17) have been used for calibration. The uncertainties are approximately $\pm 0.3 \mathrm{eV}$.

\begin{tabular}{|c|c|c|c|c|}
\hline$I(\mathrm{eV})$ & Eu II & $I(\mathrm{eV})$ & Gd II & $\begin{array}{l}\text { Core } \\
\text { hole }\end{array}$ \\
\hline $\begin{array}{l}142.5 \\
137.6\end{array}$ & $\begin{array}{l}{[\mathrm{Kr}] 4 d^{9} 5 s^{2} 5 p^{6} 4 f^{7} 6 s^{2}\left({ }^{9} D\right)} \\
{[\mathrm{Kr}] 4 d^{9} 5 s^{2} 5 p^{6} 4 f^{7} 6 s^{2}\left({ }^{9} D\right)}\end{array}$ & $\begin{array}{l}156.6 \\
151.3\end{array}$ & $\begin{array}{l}{[\mathrm{Kr}] 4 d^{9} 5 s^{2} 5 p^{6} 4 f^{7}\left({ }^{9} D\right) 5 d 6 s^{2}} \\
{[\mathrm{Kr}] 4 d^{9} 5 s^{2} 5 p^{6} 4 f^{7}\left({ }^{9} D\right) 5 d 6 s^{2}}\end{array}$ & $4 d^{-1}$ \\
\hline $\begin{array}{l}50.4 \\
46.5\end{array}$ & $\begin{array}{l}{[\mathrm{Kr}] 4 d^{10} 5 s 5 p^{6} 4 f^{7} 6 s^{2}\left({ }^{7} S\right)} \\
{[\mathrm{Kr}] 4 d^{10} 5 s 5 p^{6} 4 f^{7} 6 s^{2}\left({ }^{9} S\right)}\end{array}$ & $\begin{array}{l}55.5 \\
51.5\end{array}$ & $\begin{array}{l}{[\mathrm{Kr}] 4 d^{10} 5 s 5 p^{6} 4 f^{7}\left({ }^{7} S\right) 5 d 6 s^{2}} \\
{[\mathrm{Kr}] 4 d^{10} 5 s 5 p^{6} 4 f^{7}\left({ }^{9} S\right) 5 d 6 s^{2}}\end{array}$ & $5 s^{-1}$ \\
\hline $\begin{array}{l}38.0 \\
35.8\end{array}$ & & $\begin{array}{l}45.5 \\
43.1 \\
39.3\end{array}$ & & $5 p^{-1}$ \\
\hline $\begin{array}{l}32.5 \\
26.7\end{array}$ & $\begin{array}{l}{[\mathrm{Kr}] 4 d^{10} 5 s^{2} 5 p^{5} 4 f^{7} 6 s^{2}\left({ }^{7} P\right)} \\
{[\mathrm{Kr}] 4 d^{10} 5 s^{2} 5 p^{5} 4 f^{7} 6 s^{2}\left({ }^{9} P\right)}\end{array}$ & $\begin{array}{l}35.9 \\
29.2\end{array}$ & $\begin{array}{l}{[\mathrm{Kr}] 4 d^{10} 5 s^{2} 5 p^{5} 4 f^{7}\left({ }^{7} P\right) 5 d 6 s^{2}} \\
{[\mathrm{Kr}] 4 d^{10} 5 s^{2} 5 p^{5} 4 f^{7}\left({ }^{9} P\right) 5 d 6 s^{2}}\end{array}$ & \\
\hline $\begin{array}{l}17.5 \\
15.4 \\
13.4\end{array}$ & $\begin{array}{l}{[\mathrm{Xe}] 4 f^{6} 6 s 7 s\left({ }^{7} F\right)} \\
{[\mathrm{Xe}] 4 f^{6} 6 s^{2}\left({ }^{5} L\right)}\end{array}$ & $\begin{array}{l}25.3 \\
20.9\end{array}$ & $\begin{array}{l}{[\mathrm{Xe}] 4 f^{6}\left({ }^{7} F\right)(5 d 6 s)^{2} 7 s} \\
{[\mathrm{Xe}] 4 f^{6}\left({ }^{5} L\right) 5 d 6 s^{2}}\end{array}$ & $4 f^{-1}$ \\
\hline 10.3 & {$[\mathrm{Xe}] 4 f^{6} 6 s^{2}\left({ }^{7} F\right)$} & 17.4 & {$[\mathrm{Xe}] 4 f^{6}\left({ }^{7} F\right) 5 d 6 s^{2}$} & \\
\hline 5.8 & {$[\mathrm{Xe}] 4 f^{7} 6 s\left({ }^{7,9} S\right)$} & $\begin{array}{r}12.5 \\
10.3 \\
6.5\end{array}$ & $\begin{array}{l}{[\mathrm{Xe}] 4 f^{8}\left({ }^{7} F\right) 6 p} \\
{[\mathrm{Xe}] 4 f^{7} 5 d 6 s\left({ }^{8,10} D\right)} \\
{[\mathrm{Xe}] 4 f^{7} 6 s^{2}\left({ }^{8} S\right)}\end{array}$ & $(5 d, 6 s)^{-1}$ \\
\hline
\end{tabular}

state. ${ }^{17} \mathrm{Gd}$ and $\mathrm{Eu}$ are both distinguished by the stable $4 f^{7}\left({ }^{8} S_{7 / 2}\right)$ state of the $4 f$ shell, which explains the close similarity of the corresponding electron spectra. The interpretation of the Eu spectrum can be transferred to the Gd spectrum. The increased nuclear charge shifts all lines towards higher ionization energies. The additional line at $10.3 \mathrm{eV}$ binding energy is attributed to a $(5 d, 6 s)^{-1}$ satellite emission leaving the ion in an excited Gd II $[\mathrm{Xe}] 4 f^{8} 6 p$ state. ${ }^{17}$ The tentative assignment of the Gd electron lines is included in Table I.

$\mathrm{Sm}$ has been evaporated at a temperature of $\cong 900 \mathrm{~K}$ resulting in a population of the $\mathrm{Sm}[\mathrm{Xe}] 4 f^{6} 6 s^{2}\left({ }^{7} F_{0}\right)$ ground state and the $\mathrm{Sm}[\mathrm{Xe}] 4 f^{6} 6 s^{2}\left({ }^{7} F_{J}\right)$ excited states. Despite this and the missing of one $4 f$ electron the Sm electron spectra display the same characteristic features as the Eu and Gd spectra. This justifies an interpretation along the same lines. The splitting of the Sm II $4 f^{5} 6 s^{2}\left({ }^{6} P,{ }^{6} F,{ }^{6} H\right)$ states determines the separation of the $4 f^{-1} 6 s^{2}$ photoelectron lines $\left(E_{B}=9.2 \mathrm{eV} ; 11.9 \mathrm{eV}\right)$ and the $4 f^{-1} 6 s 7 s$ satellite lines $\left(E_{B}=16.3 \mathrm{eV} ; 19.0 \mathrm{eV}\right)$. The binding energies are in agreement with those reported by Lee et al. ${ }^{20} 5 p^{-1}$ emission gives rise to the group of lines with $25 \mathrm{eV} \leq E_{B} \leq 32 \mathrm{eV}$. The $5 p$ spin-orbit splitting and the $5 p-4 f$ interaction causes the splitting. Satellite emission contributes to the high-energy part of the group. The separation of the $5 s^{-1}$ lines is determined by the interaction of the open $5 s$ shell with the $4 f^{6}$ shell. In analogy to the findings for $\mathrm{Eu}$ we tend to attribute the splitting of the $4 d^{-1}$ lines in Fig. 2 mainly to the spinorbit interaction. Our tentative assignment of the Sm photoelectron lines is summarized in Table II. The assignment in part is based on published binding energies ${ }^{17}$ and on calculations performed within the "sudden approximation". ${ }^{21}$ The photoelectron spectra presented here confirm those published earlier. ${ }^{22}$ The interpretation presented at that time is consistent with the present interpretation.

As we will see later on the direct $4 d^{-1} \epsilon f$ channel contributes much less to the $\mathrm{Sm}, \mathrm{Eu}$, and $\mathrm{Gd} 4 d$ giant resonances than determined for $\mathrm{Ba}, \mathrm{La}$, and $\mathrm{Ce} .{ }^{1}$ Accordingly, the Auger decay of the $4 d$ hole states of the ions give rise to relatively weak structures in the electron spectra displayed in Fig. 2. Therefore we also expect multistep and double Auger decays, a major origin of the continuous background, to be less important. Indeed the back-

TABLE II. Ionization energies of atomic Sm [Xe] $4 f^{6} 6 s^{2}\left({ }^{7} F_{0, \ldots, 4}\right)$. The $6 s^{-1}$ energy from optical data (Ref. 17) has been used for calibration. The uncertainties are approximately $\pm 0.3 \mathrm{eV}$.

\begin{tabular}{rlc}
\hline$I(\mathrm{eV})$ & \multicolumn{1}{c}{ Sm II } & Core hole \\
\hline 136.8 & {$[\mathrm{Kr}] 4 d^{9} 5 s^{2} 5 p^{6} 4 f^{6} 6 s^{2}$} & $4 d^{-1}$ \\
135.5 & {$[\mathrm{Kr}] 4 d^{9} 5 s^{2} 5 p^{6} 4 f^{6} 6 s^{2}$} & \\
130.9 & {$[\mathrm{Kr}] 4 d^{9} 5 s^{2} 5 p^{6} 4 f^{6} 6 s^{2}$} & \\
48.2 & {$[\mathrm{Kr}] 4 d^{10} 5 s 5 p^{6} 4 f^{6} 6 s^{2}\left[{ }^{6} F\right]$} & $5 s^{-1}$ \\
45.2 & {$[\mathrm{Kr}] 4 d^{10} 5 s 5 p^{6} 4 f^{6} 6 s^{2}\left[{ }^{8} F\right]$} & \\
37.9 & & $5 p^{-1}$ \\
35.0 & & \\
31.4 & {$[\mathrm{Kr}] 4 d^{10} 5 s^{2} 5 p^{5} 4 f^{6} 6 s^{2}$} & \\
26.9 & {$[\mathrm{Kr}] 4 d^{10} 5 s^{2} 5 p^{5} 4 f^{6} 6 s^{2}$} & \\
25.3 & {$[\mathrm{Kr}] 4 d^{10} 5 s^{2} 5 p^{5} 4 f^{6} 6 s^{2}$} & \\
19.0 & {$[\mathrm{Xe}] 4 f^{5} 6 s 7 s\left({ }^{6} P\right)$} & \\
16.3 & {$[\mathrm{Xe}] 4 f^{5} 6 s 7 s\left({ }^{6} H,{ }^{6} F\right)$} & \\
11.9 & {$[\mathrm{Xe}] 4 f^{5} 6 s^{2}\left({ }^{6} P\right)$} & \\
9.2 & {$[\mathrm{Xe}] 4 f^{5} 6 s^{2}\left({ }^{6} H,{ }^{6} F\right)$} & $6 s^{-1}$ \\
5.7 & {$[\mathrm{Xe}] 4 f^{6} 6 s\left({ }^{6,8} F\right)$} & \\
\hline \hline
\end{tabular}


ground, indicated by the dashed lines in Fig. 2, in relation to the photoelectron lines is considerably weaker than encountered for $\mathrm{Ba}, \mathrm{La}$, and Ce. ${ }^{1}$ This finding is in good agreement with the results of recent ion yield measurements. $^{23,24}$ Whereas the giant resonances of $\mathrm{Ba}, \mathrm{La}$, and Ce decay to triply and even quadruply charged ions, the Gd $4 d \rightarrow(4, \epsilon) f$ excitation mainly results in doubly charged ions.

\section{B. Partial and total photoionization cross sections}

$$
\text { 1. Sm }
$$

The experimental total and partial cross sections of atomic Sm are displayed in Fig. 3. The partial cross sections comprise the intensity of the main photoelectron lines and the satellite lines (see Table II). Compared to

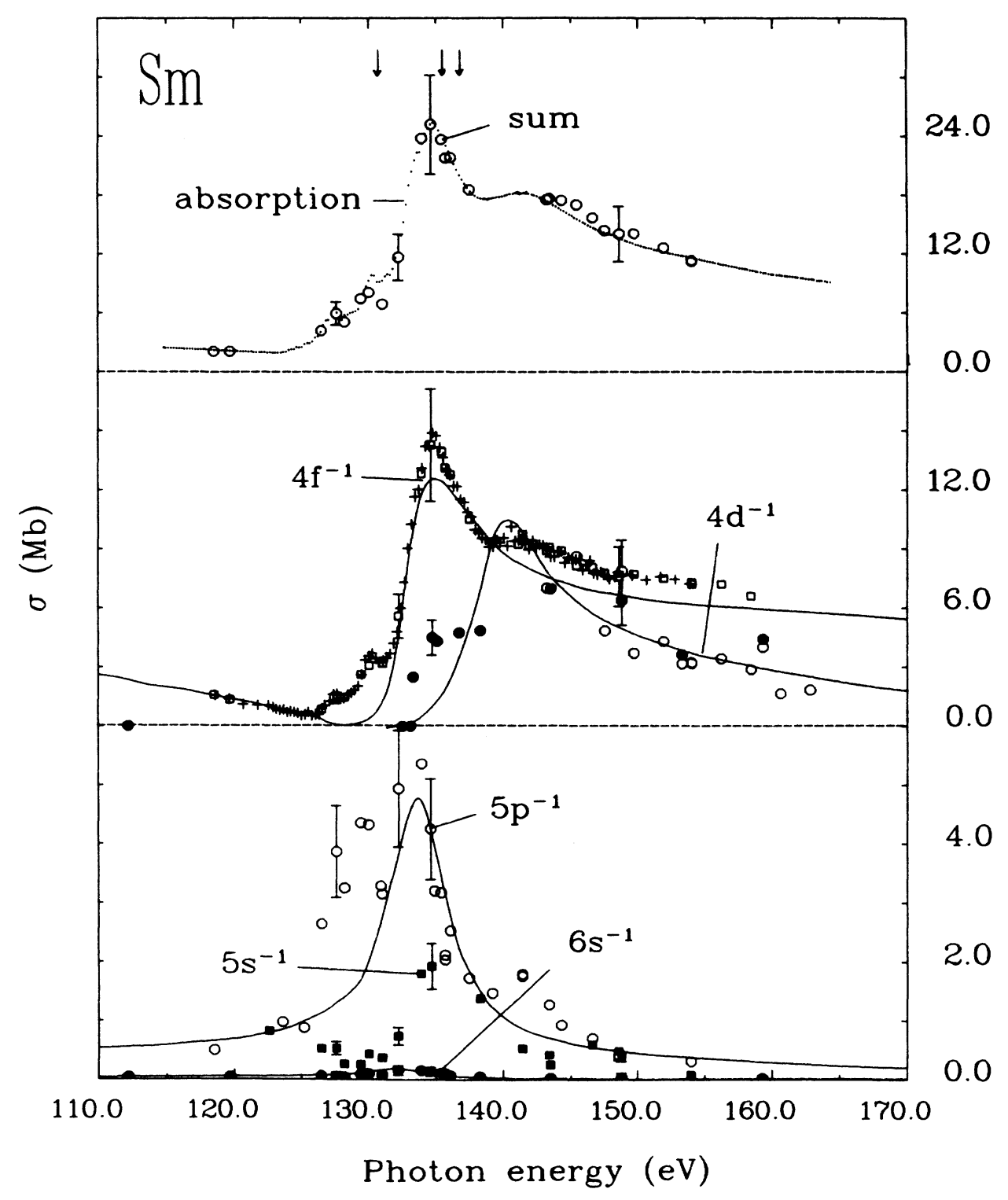

FIG. 3. Top: Experimental photoabsorption cross section ( $\cdots)$ (Ref. 13) and the sum of all partial photoionization cross sections $(\bigcirc \bigcirc \bigcirc)$ for atomic Sm. The arrows mark the position of the $4 d^{-1}$ ionization limits determined from the main photoelectron lines. Center: Sm $4 d^{-1}$ partial cross section determined from the photoelectron lines $(\circ \circ \bigcirc)$ and the Auger lines $(\infty)$ ) together with the $4 f^{-1}$ partial cross section ( $\square \square$, CIS: +++ ). The results of RTDLDA calculations $(-)$ (Ref. 9) are included. The calculated $4 d^{-1}$ cross section has been multiplied by a factor of 0.5 and shifted by $6 \mathrm{eV}$ towards higher photon energies. Bottom: Experimental $5 s^{-1}(\mathbb{D}), 5 p^{-1}(\bigcirc \bigcirc \bigcirc)$, and $6 s^{-1}(\omega)$ partial cross sections of atomic Sm. The corresponding RTDLDA calculations ( - ) (Ref. 9) are given for comparison. The theoretical $4 f^{-1}, 5 p^{-1}$, and $6 s^{-1}$ cross sections have been shifted by $1 \mathrm{eV}$ towards higher photon energies. 
the earlier results ${ }^{22,25,26}$ the new results represent a considerable improvement. The $4 f^{-1}$ cross section has been determined from a constant-ionic-state spectrum (CIS), the $4 d^{-1}$ cross section has been extended towards lower energies, and the $5 s^{-1}$ and $6 s^{-1}$ cross sections have also been determined. The sum of all partial cross sections displayed in the bottom and center part of Fig. 3 reproduces the energy dependence of the absorption cross section very well. ${ }^{13}$ The absorption spectrum given in the top part of Fig. 3 has been normalized to the sum of the experimental partial cross sections at the low-energy end and at the maximum at $h v=134 \mathrm{eV}$. The normalization at the low-energy end eliminates the uncertainties due to the considerable errors involved in positioning the absorption spectra relative to the origin of the abscissa. ${ }^{13}$ Only relative cross sections could be determined in our experiments. The absolute scale in Fig. 3 is based on the normalization of the experimental $4 f^{-1}$ partial cross section to the cross section predicted by RTDLDA calculations. ${ }^{9}$ The theoretical $4 f^{-1}$ cross section, included in the center part of Fig. 3, has been shifted by $1 \mathrm{eV}$ towards higher photon energies. The $4 d^{-1}$ partial cross section has been determined directly from the $4 d^{-1}$ photoelectron lines and indirectly from the $\mathrm{N}_{45} \mathrm{O}_{23} \mathrm{~N}_{67}$ Auger lines. By multiplying the latter values by a factor of 1.3 both sets of data have been matched in the region of overlap. For photon energies between $138 \mathrm{eV}$ and $143 \mathrm{eV}$ the Auger lines merge with the $5 s^{-1}$ photoelectron lines and therefore no reliable $4 d^{-1}$ cross section could be determined.

The character of the Sm giant resonance definitely differs from that of a shape resonance encountered for $\mathrm{Ba}, \mathrm{La}$, and $\mathrm{Ce}$. The $\mathrm{Sm} 4 f^{-1}, 5 p^{-1}$, and $5 s^{-1}$ autoionization channels comprise considerably more oscillator strengths than the direct $4 d^{-1}$ ionization channel, whereas for $\mathrm{Ba}, \mathrm{La}$, and $\mathrm{Ce}$ it is quite the reverse; the $4 d^{-1}$ ionization channel is by far the strongest. The maximum of the $\mathrm{Sm}$ resonance lies below the thresholds of two of the strongest $4 d^{-1}$ ionization continua. For Ba, $\mathrm{La}$, and $\mathrm{Ce}$ the resonances peak above the corresponding thresholds. ${ }^{1}$ The Sm resonances located below $h v=140$ $\mathrm{eV}$ can be well characterized as autoionizing $4 d^{9} 4 f^{7}$ excitations split by spin-orbit and $4 d-4 f$ interactions. Especially the low-energy resonances are strongly coupled to the $5 p^{-1}$ ionization channel (see bottom part of Fig. 3). The direct $4 d^{-1}$ ionization cross section peaks $\cong 7 \mathrm{eV}$ above the main resonance. The photon energy dependence of the $4 d^{-1}$ partial cross section resembles that of a shape resonance. There are two significant discrepancies between the results of the RTDLDA calculations 9 and the experimental results. Compared with the $4 f^{-1}$ cross section the theory overestimates the $4 d^{-1}$ cross section by a factor of 2 and places the maximum too close to the $4 f^{-1}$ resonance. The theoretical $4 d^{-1}$ spectrum given in the center part of Fig. 3 has been multiplied by a factor of 0.5 and shifted towards higher photon energies by $6 \mathrm{eV}$. This spectrum reproduces the experimental spectrum fairly well. Shifting the calculated $5 p^{-1}$ and $6 s^{-1}$ partial cross sections towards higher energies by 1 $\mathrm{eV}$ results in a reasonable agreement between experiment and theory. There is no obvious way how the wrong theoretical partition of the oscillator strengths can be remedied. Therefore, we consider the above normalization to the strongest partial cross section the safest. But we are well aware of the probability that the absolute cross sections obtained that way are too low, because part of the theoretical $4 d^{-1}$ cross section has been omitted. The RTDLDA calculations are not expected to repro-

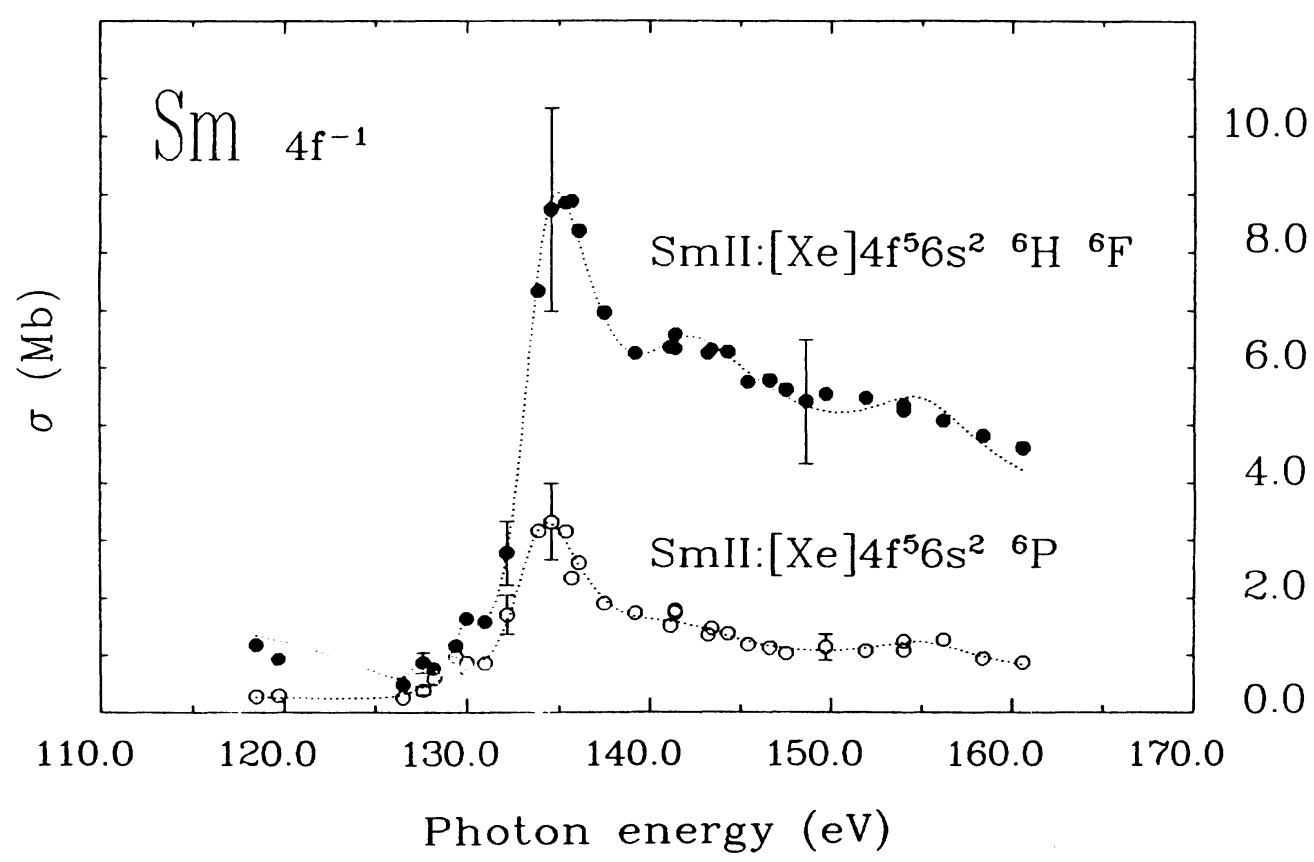

FIG. 4. Partial photoionization cross sections of the $4 f^{-1}$ main photoelectron lines for atomic Sm. The dotted lines represent an approximation to the data points by a superposition of Fano-type profiles. 
duce the fine structure. Therefore the resonances at $127.5 \mathrm{eV}, 129.6 \mathrm{eV}$, and $141 \mathrm{eV}$ are missing in the calculated cross sections.

The partial cross sections of the two main $4 f^{-1}$ photoelectron lines (see Fig. 2 and Table II) are depicted separately in Fig. 4. The experimental data have been approximated by a superposition of Fano-type profiles: ${ }^{27-33}$

$$
\sigma_{P}(h v)=\sigma_{b, P}+\sum_{n} \sigma_{a, P, n} \frac{\left[q_{P, n}+\frac{h v-h v_{n}}{\Gamma_{n} / 2}\right]^{2}}{1+\left(\frac{h v-h v_{n}}{\Gamma_{n} / 2}\right)^{2}} .
$$

$P$ denotes the photoionization channel. The sum extends over all resonances $n$ characterized by a resonance energy $h v_{n}$ and a half-width $\Gamma_{n}$. The asymmetry parameters $q_{P, n}$ and the interacting cross section $\sigma_{a, P, n}$ depend on the ionization channel and the resonance. The noninteracting background is given by $\sigma_{b, P}$. In the case of many interacting resonances and continua this parametrization represents a considerable simplification and therefore should be considered as an empirical method to describe the spectra. The parameters used in the calculation of the curves presented in Fig. 4 are listed in Table III. The resonance energies $127.5 \mathrm{eV}, 129.6 \mathrm{eV}, 133.8 \mathrm{eV}$, and $141 \mathrm{eV}$ agree with energies obtained in Hartree plus statistical exchange (HX) calculations ${ }^{34,35}$ of the $4 d^{10} 4 f^{6}\left({ }^{7} F_{0}\right) \rightarrow 4 d^{9} 4 f^{7}$ excitations in intermediate coupling using the Cowan code. ${ }^{35}$ Double excitation processes possibly contribute to the maximum at $156 \mathrm{eV}$.

In summarizing our results, we can state that the lowenergy resonances are predominantly of $4 d^{9} 4 f^{7}$ character whereas towards higher photon energies $4 d-\epsilon f$ transitions constitute a major part of the $4 d$ excitations. Upon solidification $\mathrm{Sm}$ changes its ground-state configuration to $[\mathrm{Xe}] 4 f^{5}(5 d 6 s)^{3} .^{36-38}$ Only the surface atoms preserve the atomic $[\mathrm{Xe}] 4 f^{6} 6 s^{2}$ configuration and display resonance profiles similar to those of the free atoms. ${ }^{39}$

\section{2. $E u$}

The half-filled $4 f$ shell of the $\mathrm{Eu}[\mathrm{Xe}] 4 f^{7} 6 s^{2}\left({ }^{8} S_{7 / 2}\right)$ ground state and its quasiclosed-shell character have made the Eu $4 d$ resonance the object of several experimental $^{25,40}$ and theoretical ${ }^{8-11}$ studies. The experimental absorption spectrum ${ }^{12}$ and our total and partial cross sections are given in Fig. 5. The partial cross sections comprise the intensity of the main photoelectron lines and the satellite lines (see Table $\mathrm{I}$ ). The results obtained by RTDLDA, ${ }^{9}$ RPAE, ${ }^{10}$ and MBPT (Ref. 11) calculations are included in Fig. 5. The RPAE cross section has been calculated by using the Fano parameters given in the publication. ${ }^{10}$ Only relative cross sections could be determined in the experiments. By normalizing the experimental $4 f^{-1}$ cross section to the theoretical cross sections $^{9,11}$ in the energy range above the resonance, the absolute scale for the cross sections has been established. For the same reasons as discussed for $\mathrm{Sm}$ the absorption spectrum has been normalized to the sum of the partial cross sections at the maximum and at the high-energy end. Except for photon energies below $125 \mathrm{eV}$ this results in a good agreement of both spectra. The maximum of the resonance lies between the spin-orbit split $4 d^{-1}\left({ }^{9} D\right)$ ionization thresholds. The RTDLDA calculation $^{9}$ reproduces the asymmetric profile of the resonance but places the maximum at a too low energy. The absorption spectrum calculated by Amusia, Sheftel, and Chernysheva ${ }^{10}$ describes the experimental spectrum quite well. The RPAE calculations ${ }^{10}$ are based on the assumption that the resonance is determined by $4 d^{10} 4 f^{7}\left({ }^{8} S\right) \rightarrow 4 d^{9} 4 f^{8} \quad$ excitations and $4 d^{9} 4 f^{8}$ $\rightarrow 4 d^{10} 4 f^{6} \epsilon g$ autoionization decays. Our partial cross sections, displayed in the center and bottom part of Fig. 5 , corroborate that the autoionization into the $4 f^{-1} \epsilon l$ continuum prevails by far. The $4 f^{-1}$ partial cross section comprises more than two times the oscillator strength of the $4 d^{-1}$ cross section. The latter almost exclusively has been determined from the $\mathrm{N}_{45} \mathrm{O}_{23} \mathrm{~N}_{67}$ Auger lines. The $4 d^{-1}$ photoelectron lines could only be evaluated for photon energies more than $10 \mathrm{eV}$ above the resonance. The maximum of the $4 d^{-1}$ cross section is close to that of the $4 f^{-1}$ cross section. The MBPT $4 d^{-1}$ and $4 f^{-1}$ partial cross sections ${ }^{11}$ are in fair agreement with the experimental results. The $\operatorname{RTDLDA}^{9} 4 f^{-1}$ and $4 d^{-1}$ partial cross sections almost coincide. The theory overestimates the $4 d^{-1}$ cross section by more than a factor of 2. The $\operatorname{RTDLDA}^{9} 4 f^{-1}$ cross section is close to the experimental spectrum, the main differences being the too large half width and the too low peak position predicted by the theory.

The maximum of the $5 p^{-1}$ cross section located at 140 $\mathrm{eV}$ is well described by the MBPT $^{11}$ calculations. Zangwill and Doolen ${ }^{9}$ obtained a very similar resonance only shifted towards lower energies by $2 \mathrm{eV}$. None of the theories correctly reproduces the fine structure in the energy range 132 to $137 \mathrm{eV}$ and the coupling of these excitations to the $4 f^{-1}, 5 p^{-1}$ and $5 s^{-1}$ ionization channels.

TABLE III. Parameters determined by approximating the Sm spectra in Fig. 4 by a superposition of Fano profiles according to Eq. (1) with $\sigma_{b,{ }^{6}(H, F)}=0.00 \mathrm{Mb}$ and $\sigma_{b_{2},{ }_{P}}=0.21 \mathrm{Mb}$.

\begin{tabular}{cccccccc}
\hline & & \multicolumn{2}{c}{ SmII $[\mathrm{Xe}] 4 f^{5} 6 s\left({ }^{6} H,{ }^{6} \mathrm{~F}\right)$} & \multicolumn{2}{c}{ SmII $[\mathrm{Xe}] 4 f^{5} 6 s^{2}\left({ }^{6} P\right)$} \\
$\sigma_{a} q^{2}(\mathrm{Mb})$ & $\boldsymbol{\sigma}_{a} q^{2}(\mathrm{Mb})$ \\
\hline 1 & 127.5 & 1.8 & 4.0 & 0.63 & $q$ & 0.08 \\
2 & 129.6 & 1.8 & 2.0 & 1.02 & $\infty$ & 0.59 \\
3 & 133.8 & 4.6 & 2.0 & 6.76 & $\infty$ & 2.91 \\
4 & 141 & 8 & 1.6 & 1.71 & 3.9 & 0.47 \\
5 & 156 & 8 & $\infty$ & 1.35 & $\infty$ & 0.48 \\
\hline \hline
\end{tabular}




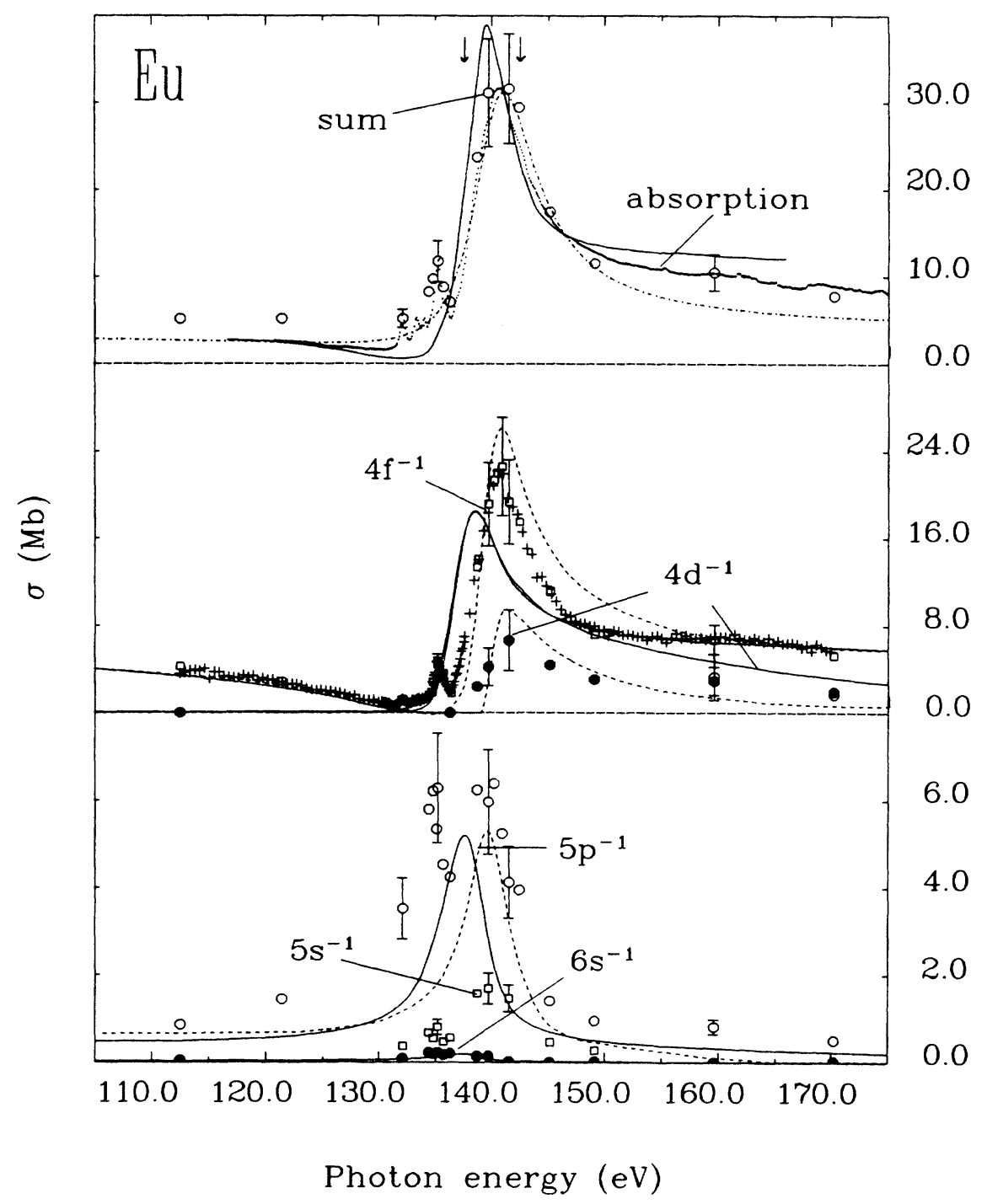

FIG. 5. Top: Experimental photoabsorption cross section (...) (Ref. 12) and theoretical photoionization cross sections, RTDLDA ( -) (Ref. 9) and RPAE (_- -.-.) (Ref. 10) of atomic Eu. The sum of the partial photoionization cross sections is given by the open circles. The arrows mark the position of the $4 d^{-1}$ photoionization limits determined from the main photoelectron lines. Center: $4 d^{-1}$ partial cross section determined from the photoelectron lines $(\bigcirc \bigcirc \bigcirc)$ and the Auger lines $(\hookrightarrow \infty)$ together with the $4 f^{-1}$ partial cross section ( $\square \square$, CIS: +++ ). The results of RTDLDA $(-)$ (Ref. 9) and MBPT (- - ) (Ref. 11) are included. Bottom: Experimental $5 s^{-1}(\square \square), 5 p^{-1}(\bigcirc \bigcirc \bigcirc)$, and $6 s^{-1}(\omega)$ partial cross sections of atomic Eu. The corresponding RTDLDA ( - ) (Ref. 9) and MBPT (- - ) (Ref. 11) calculations are given for comparison.

Both the $4 f^{-1}$ and the $5 p^{-1}$ cross sections display a prominent resonance at $135 \mathrm{eV}$. Within the error bars our results are in good agreement with the partial cross sections and the electron yield spectrum obtained by Becker et al. 40,41 $^{2}$

The $4 f^{7} 6 s^{2}\left({ }^{8} S\right) \rightarrow\left(4 f^{6} 6 s^{2}\left({ }^{7} F\right), 4 f^{6} 6 s 7 s\left({ }^{7} F\right), \quad\right.$ and $\left.4 f^{6} 6 s^{2}\left({ }^{5} L\right)\right) \epsilon l$ partial cross sections are separately plotted in Fig. 6. All experimental spectra have been approximated by a superposition of Fano-type profiles as de- scribed by Eq. 1. The parameters used are listed in Table IV. In all three spectra the main resonance can be described by a single Fano-type profile with the same resonance energy and half width and almost the same asymmetry parameter $q$. The resonance at $135 \mathrm{eV}$ only gives rise to, in relation to the main resonance, a weak and almost symmetric line in the $4 f^{-1}$ ionization channels leaving the ion in either the $4 f^{6} 6 s^{2}\left({ }^{7} F\right)$ or the $4 f^{6} 6 s 7 s\left({ }^{7} F\right)$ state. In contrast to this in the spectrum of 


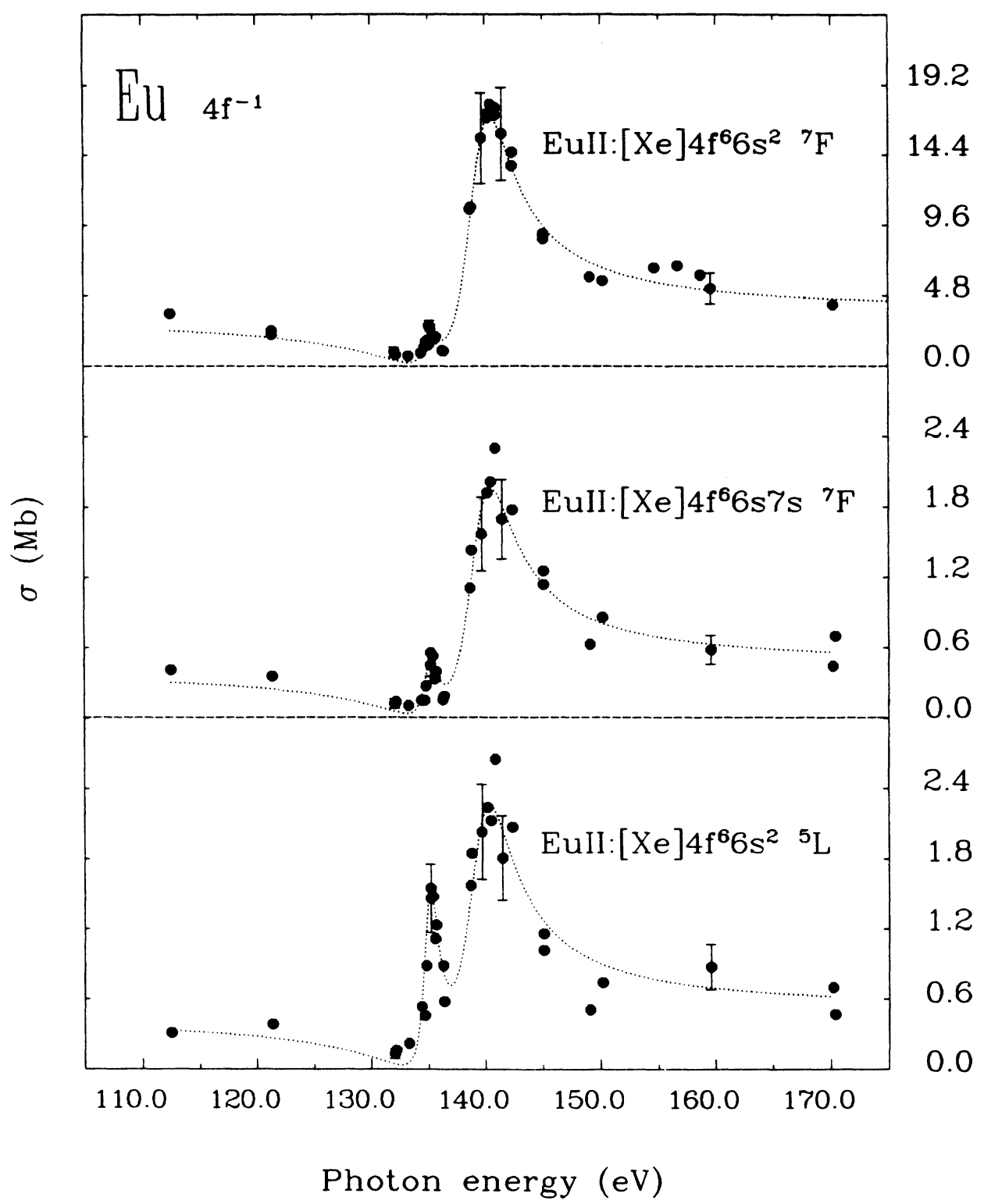

FIG. 6. Partial photoionization cross section of different $4 f^{-1}$ processes for atomic Eu. The dotted lines represent the approximation of the data points by a superposition of Fano-type profiles.

the $4 f^{7} 6 s^{2}\left({ }^{8} S\right) \rightarrow 4 f^{6} 6 s^{2}\left({ }^{5} L\right)$ satellites the strength of this resonance becomes comparable to the strength of the main resonance. The assignment of this satellite is tentative and therefore it is interesting to note, that $\mathrm{HX}$ calculations $^{34}$ ascribe a considerable $4 d^{10} 4 f^{7}\left({ }^{8} S\right)$ $\rightarrow 4 d^{9}\left(4 f^{85} D\right)$ character to the resonance at $135 \mathrm{eV}$ whereas the main resonance is predominantly caused by $4 d^{10} 4 f^{7}\left({ }^{8} S\right) \rightarrow 4 d^{9}\left(4 f^{8}\left({ }^{7} F\right)\right)$ transitions. This favors the assignment of the satellite to an $4 f^{6}\left({ }^{5} L\right) 6 s^{2}$ ion state. A recoupling of the $4 f$ electrons has also been invoked for

TABLE IV. Parameters determined by approximating the Eu spectra in Fig. 6 by a superposition of Fano profiles according to Eq. (1) with $\sigma_{b, P}=0$.

\begin{tabular}{ccccccccc}
\hline \hline & $h v_{n}$ & $\Gamma_{n}$ & \multicolumn{2}{c}{ Eu II $[\mathrm{Xe}] 4 f^{6} 6 s^{2}\left({ }^{7} F\right)$} & \multicolumn{2}{c}{ Eu II } & {$[\mathrm{Xe}] 4 f^{6} 6 s 7 s\left({ }^{7} F\right)$} & \multicolumn{2}{c}{ Eu II $[\mathrm{Xe}] 4 f^{6} 6 s^{2}\left({ }^{5} L\right)$} \\
$n$ & $(\mathrm{eV})$ & $(\mathrm{eV})$ & $q$ & $\sigma_{a} q^{2}(\mathrm{Mb})$ & $q$ & $\sigma_{a} q^{2}(\mathrm{Mb})$ & $q$ & $\sigma_{a} q^{2}(\mathrm{Mb})$ \\
\hline 1 & 135.0 & 1.5 & 4.0 & 2.29 & 4.0 & 0.47 & 4.0 & 1.45 \\
2 & 139.3 & 4.6 & 2.0 & 13.46 & 1.9 & 1.48 & 2.1 & 1.72 \\
\hline \hline
\end{tabular}


the explanation of a satellite line in the photoelectron spectrum of solid Gd by Gerken, Barth, and Kunz. ${ }^{18}$ But there are still questions because Pan, Carter, and Kelly ${ }^{11}$ ascribed the small peak at $134.5 \mathrm{eV}$ in their $4 f^{-1}$ spectrum to the $4 d^{10} 4 f^{7}\left({ }^{8} S\right) 6 s^{2}$ $\rightarrow 4 d^{9} 4 f^{7}\left({ }^{9} D\right) 6 s^{2} 6 p$ transition.

The photoionization cross sections of solid Eu (Refs. 39,42 ) with regard to the relative amplitudes differ from our results on the free atoms. In comparison to the $4 f^{-1}$ cross section the $4 d^{-1}$ ionization is stronger and the $5 p^{-1}$ channel is weaker in the metal. The solid-state spectra agree better with the results of the RTDLDA cal- culations especially in respect to the $\sigma(4 f) / \sigma(4 d)$ ratio. Zangwill ${ }^{8}$ attributes the discrepancy between the experimental and the theoretical spectra of the free atoms to the spherical averaging performed in the RTDLDA. Solid-state effects seem to suppress open-shell effects and thus reduce the errors due to the spherical averaging over the sublevels of all open shells.

\section{3. $\boldsymbol{G d}$}

In the ground state of $\mathrm{Gd}$ the $4 f^{7}\left({ }^{8} S_{7 / 2}\right)$ configuration is preserved. The additional electron occupies a $5 d$ state.

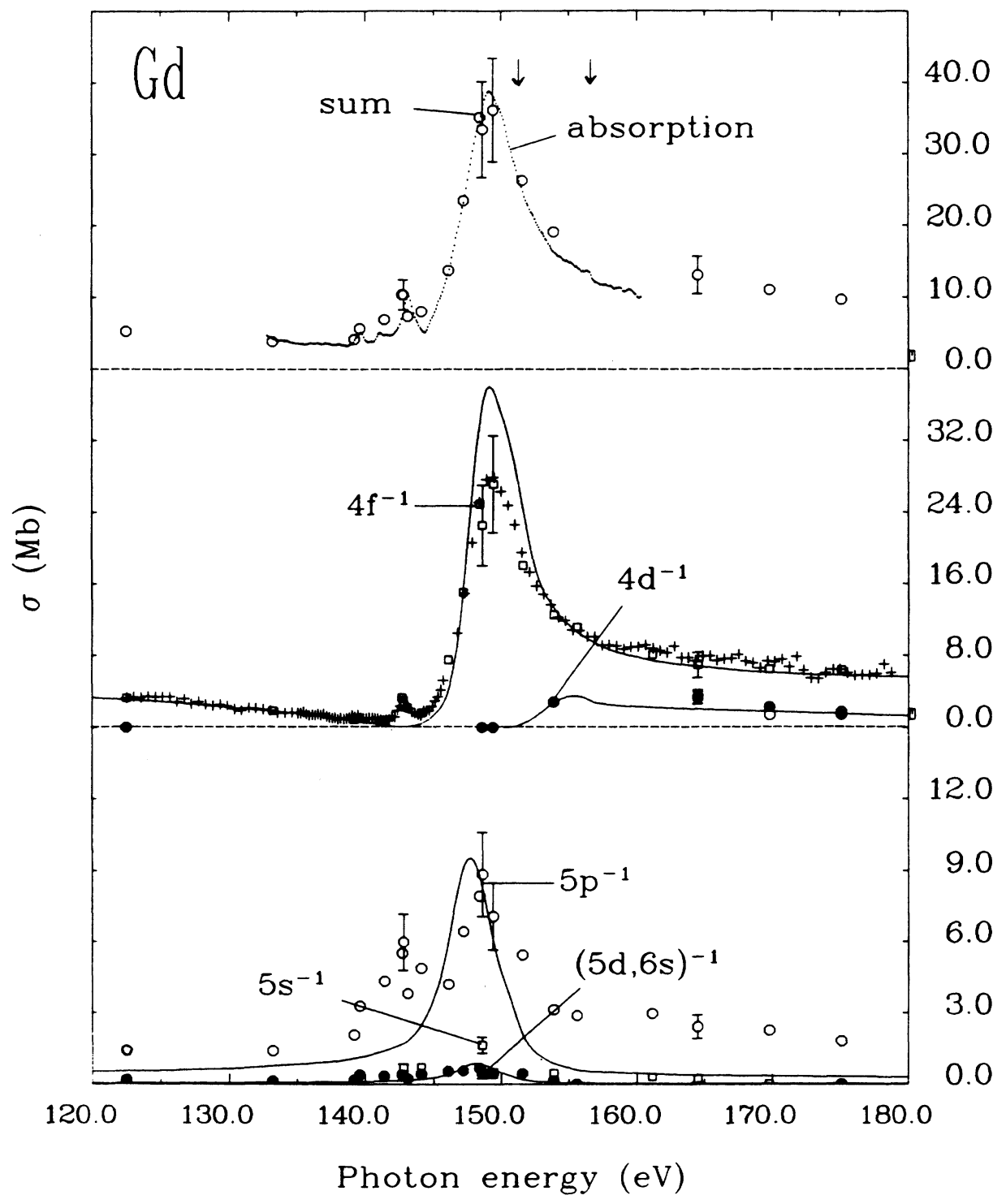

FIG. 7. Top: Experimental photoabsorption cross section (. . . .) (Ref. 14) and the sum of all partial photoionization cross sections $(\bigcirc \bigcirc \bigcirc)$ for atomic Gd. The arrows mark the position of the $4 d^{-1}$ ionization limits determined from the main photoelectron lines. Center: $\mathrm{Gd} 4 d^{-1}$ partial cross section determined from the photoelectron lines $(\bigcirc \bigcirc \bigcirc)$ and the Auger lines $(\bullet \bullet)$ together with the $4 f^{-1}$ partial cross section ( $\square \square$, CIS: +++ ). The results of RTDLDA calculations $(-$ ) (Ref. 9) are included. The calculated $4 d^{-1}$ cross section has been multiplied by a factor of 0.5 and shifted by $6 \mathrm{eV}$ towards higher photon energies. Bottom: Experimental $5 s^{-1}(\square \square), 5 p^{-1}(\bigcirc \bigcirc \bigcirc)$, and $(5 d, 6 s)^{-1}(\omega)$ partial cross sections of atomic Gd. The corresponding RTDLDA calculations ( - ) (Ref. 9) are given for comparison. The theoretical $4 f^{-1}, 5 p^{-1}$, and $(5 d, 6 s)^{-1}$ cross sections have been shifted by $2 \mathrm{eV}$ towards higher photon energies. 
Therefore the similarity of the Eu and Gd $4 d$ absorption spectra (see Fig. 1) is not surprising. Also the total and partial cross sections of Gd given in Fig. 7 display the same characteristic features as the corresponding $\mathrm{Eu}$ spectra in Fig. 5. As for Sm and Eu the absorption spectrum ${ }^{14}$ has been normalized to the sum of the partial $4 f^{-1}, 4 d^{-1}, 5 p^{-1}, 5 s^{-1}$, and $(5 d, 6 s)^{-1}$ cross sections. Both curves are given in the top part of Fig. 7. The normalization results are in good agreement between both spectra below and in the resonance. Above the resonance the absorption falls somewhat below the total photoelectron cross section, but the deviations are still within the error limits. Gd is the first element along the rare-earth series for which the $4 d$ giant resonance is located below the $4 d^{-1}$ ionization thresholds determined from the strongest $4 d^{-1}$ photoelectron lines. As demonstrated in the center part of Fig. 7 the direct $4 d^{-1} \epsilon f$ ionization only contributes a very minor part of the resonance. The $4 d^{-1}$ partial cross section has been derived from the $N_{45} O_{23} N_{67}$ Auger lines and the $4 d^{-1}$ photoelectron lines. The giant resonance is almost exclusively due to $4 d^{10} 4 f^{7} \rightarrow 4 d^{9} 4 f^{8}$ transitions strongly coupled to the $4 d^{10} 4 f^{6} \epsilon l$ continuum. Most of the oscillator strength is concentrated in the $4 f^{-1}$ channel, the $5 p^{-1}, 5 s^{-1}$, and $(5 d, 6 s)^{-1}$ channels only contain a minor fraction (center and bottom part of Fig. 7). Recent ion yield measure-

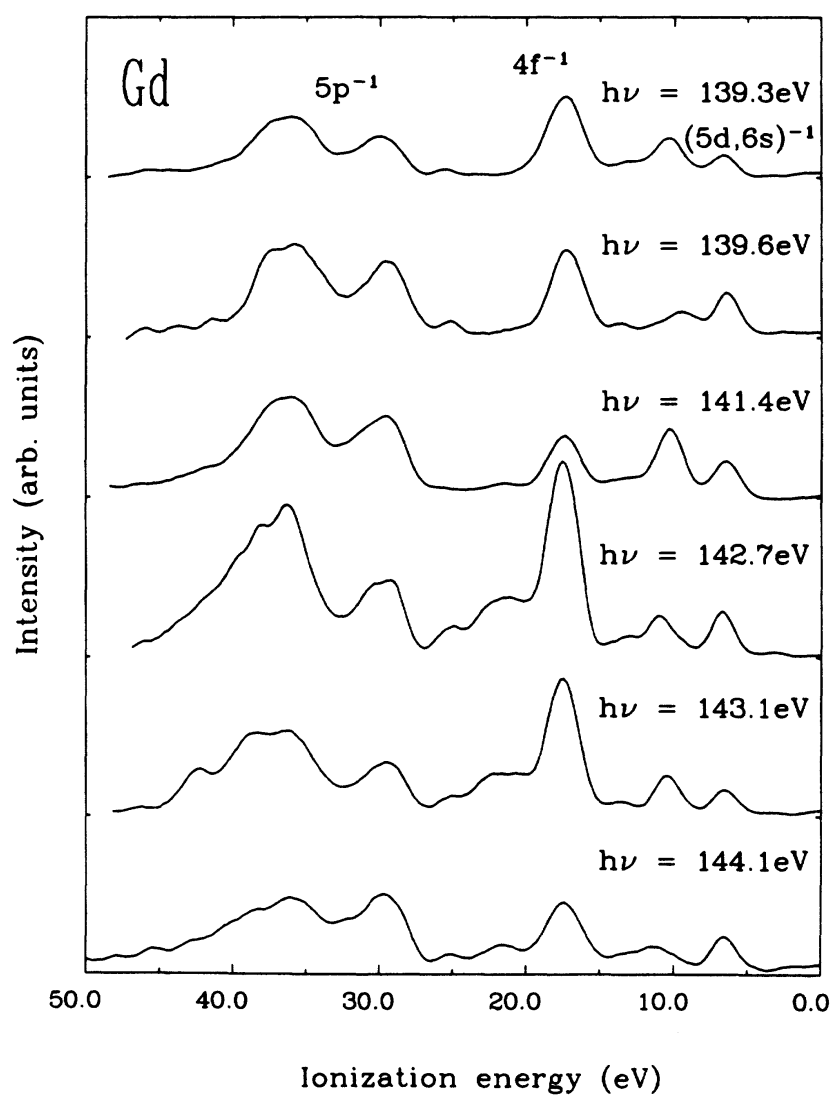

FIG. 8. Photoelectron spectra of atomic Gd taken at photon energies in the range of the resonance fine structure below the giant resonance. ments $^{24}$ show that photoabsorption in the giant resonance mainly results in doubly charged Gd ions. Multistep decays of the outer $5 p^{-1}$ and $4 f^{-1}$ hole states are far less probable than of $4 d^{-1}$ hole states generated by resonantly enhanced $4 d$ ionization as encountered for Ba, $\mathrm{La}$, and $\mathrm{Ce}$. Only relative cross sections could be determined in our experiments. As for $\mathrm{Sm}$ the absolute scale has been established by normalizing the experimental $4 f^{-1}$ cross section to the cross section obtained by RTDLDA calculations. ${ }^{9}$ In order to achieve best agreement between theory and experiment the theoretical $4 f^{-1}, 5 p^{-1}$, and $(5 d, 6 s)^{-1}$ cross sections have been shifted towards higher photon energies by $2 \mathrm{eV}$. The theoretical $4 d^{-1}$ cross section has been shifted towards higher energies by $6 \mathrm{eV}$ and multiplied by a factor of 0.5 . The resulting theoretical cross sections are very close to the experimental $4 f^{-1}$ and $4 d^{-1}$ spectra. Only in the maximum the theory overshoots the experimental $4 f^{-1}$ data. The $(5 d, 6 s)^{-1}$ channel is also well described by the theory. The agreement is less satisfactory for the $5 p^{-1}$ cross section. The theory reproduces the main resonance but falls considerably below the experimental data above the resonance. As discussed for Sm the absolute cross sections determined by the above normalization are probably too low, because of the neglect of part of the theoretical $4 d^{-1}$ cross section. Again, as for $\mathrm{Sm}$ and Eu the theory is not expected to describe the smaller resonances below the main resonance.

Figure 8 displays several photoelectron spectra taken at photon energies between 139 and $145 \mathrm{eV}$, i.e., in the region of the small absorption lines preceding the main resonance. The resonant enhancement of the various photoelectron main and satellite lines listed in Table I can be seen very easily. The satellite at $10.3 \mathrm{eV}$ binding energy tentatively assigned to a $4 f^{8}\left({ }^{7} F\right) 6 p$ final state of the ion reaches its highest amplitude at $h v=141.4 \mathrm{eV}$. The $4 f$

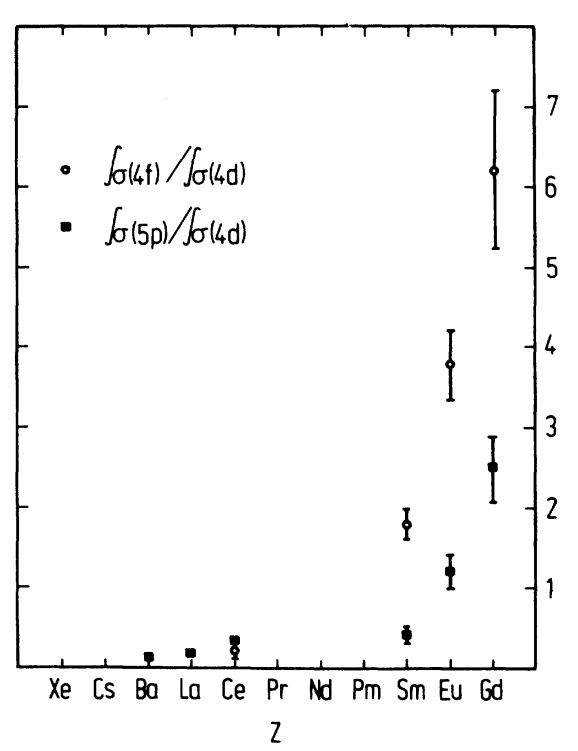

FIG. 9. Ratio of the partial cross sections integrated over the resonance $\int \sigma(4 f) / \int \sigma(4 d) ; \int \sigma(5 p) / \int \sigma(4 d)$ as a function of nuclear charge $Z$. 
satellites at binding energies of 20.9 and $25.3 \mathrm{eV}$ display the strongest enhancement at $142.7 \mathrm{eV}$. Also at this photon energy between binding energies of 40 and $45 \mathrm{eV}$ a group of $5 p^{-1}$ satellites reaches its maximal strength. The counterpart to the $4 f^{6}\left({ }^{5} L\right) 5 d 6 s^{2}$ satellite has also been detected in the spectrum of solid Gd. ${ }^{18}$ Like for the analogous case in $\mathrm{Eu} 4 d^{10} 4 f^{7}\left({ }^{8} S\right) \rightarrow 4 d^{9} 4 f^{8}\left({ }^{5} L\right)$ transitions are expected to contribute to the resonance at 142.7 $\mathrm{eV}$.

\section{C. $Z$ dependence of the giant resonance}

For $\mathrm{Ba}$ and $\mathrm{La}$ the giant resonance peaks well above the $4 d^{-1}$ ionization limits (see Fig. 1). With increasing nuclear charge $Z$ the maximum moves closer to the thresholds and for Gd it is located below the thresholds of the strongest ionization continua.

The $4 d^{-1}$ ionization dominates for low- $Z$ elements. Starting with Ce the $4 f^{-1}$ ionization gains in strength, by far outweighing the $4 d^{-1}$ ionization for $\mathrm{Gd}$. The ratio of the partial cross sections integrated over the resonance $\int \sigma(4 f) / \int \sigma(4 d)$ plotted in Fig. 9 shows a strong increase with $Z$. Also the $\int \sigma(5 p) / \int \sigma(4 d)$ ratio displays a marked increase with $Z$. The $4 f^{-1}$ and $5 p^{-1}$ cross sections peak in the energy region close to the $4 d^{-1}$ ionization thresholds, whereas the $4 d^{-1}$ cross section reaches its maximum at higher photon energies.

The character of the giant resonances changes from a
$4 d^{-1} \epsilon f$ continuum resonance in $\mathrm{Ba}$ to a bound $4 d^{-1} 4 f$ resonance in Gd. For the elements inbetween the resonance is of intermediate $4 d^{-1}(4, \epsilon) f$ character. The multiplet splitting is the origin for most of the structures displayed by the spectra. The structures superimposed on the $\mathrm{Ba}$ continuum resonance are due to multielectron excitations.

Calculations for the whole series have only been performed within the local density approximation. ${ }^{9,43}$ Many of the characteristic features and trends are reasonably well described by the theory. Deviations in peak positions and theoretical $4 d^{-1}$ cross sections which compared to the $4 f^{-1}$ cross section are approximately two times higher than the experimental one are the main discrepancies encountered between theory and experiment.

\section{ACKNOWLEDGMENTS}

The authors wish to thank the BESSY and the HASYLAB staff for continuous assistance and Gary Doolen (Los Alamos Laboratory) and Andrew Zangwill (Georgia Institute of Technology) for providing us with their unpublished RTDLDA results. We also thank Uwe Becker for discussions and for sending us results prior to publication. The financial support of the Bundeministerium für Forschung und Technologie der Bundesrepublik Deutschland is gratefully acknowledged.
"Present address: J. D. Möller Optische Werke G.m.b.H., Rosengarten 10, D-2000 Wedel, Federal Republic of Germany.

${ }^{\dagger}$ Present address: Firma CAS, Bergstrasse 8, D-2057 Reinbek, Federal Republic of Germany.

${ }^{1}$ M. Richter, M. Meyer, M. Pahler, T. Prescher, E. v. Raven, B. Sonntag, and H.-E. Wetzel, Phys. Rev. A 39, 5666 (1989).

${ }^{2}$ Giant Resonances in Atoms, Molecules, and Solids, edited by $\mathrm{J}$. P. Connerade, J. M. Esteva, and R. C. Karnatak (Plenum, New York, 1987).

${ }^{3}$ J. F. Herbst, Phys. Rev. B 28, 4204 (1983).

${ }^{4}$ B. Johansson and M. Martensson, Phys. Rev. B 21, 4427 (1980).

${ }^{5}$ M. Richter, T. Prescher, M. Meyer, E. v. Raven, B. Sonntag, H.-E. Wetzel, and S. Aksela, Phys. Rev. B 38, 1763 (1988).

${ }^{6}$ M. Lübcke, B. Sonntag, W. Niemann, and P. Rabe, Phys. Rev. B 34, 5184 (1986).

${ }^{7}$ F. Gerken, J. Barth, A. S. Flodström, L. I. Johansson, and C. Kunz, Phys. Scr. 32, 43 (1985).

${ }^{8}$ A. Zangwill, J. Phys. C. 20, L627 (1988).

${ }^{9}$ A. Zangwill and G. Doolen (unpublished).

${ }^{10}$ M. Ya. Amusia, S. I. Sheftel, and L. V. Chernysheva, Zh. Tekh. Fiz. 51, 2441 (1981) [Sov. Phys. Tech. Phys. 26, 1444 (1981)].

${ }^{11}$ C. Pan, S. L. Carter, and H. P. Kelly, J. Phys. B 20, L335 (1987).

${ }^{12}$ E. v. Raven, Diplomarbeit Universität Hamburg, Hamburg, Germany (1986) [also available as Internal Report DESYF41-86/05, Hamburg (1986)].

${ }^{13}$ H. J. Hellberg, Diplomarbeit Universität Hamburg, Hamburg,
Germany (1986).

${ }^{14}$ M. Pahler, Diplomarbeit Universität Hamburg, Hamburg, Germany (1987).

${ }^{15}$ M. Flöter, Diplomarbeit Universität Hamburg, Hamburg, Germany (1987).

${ }^{16}$ D. Handschuh, Diplomarbeit Universtität Hamburg, Hamburg, Germany (1987).

${ }^{17}$ W. C. Martin, R. Zalubas, and L. Hagan, Atomic Energy Levels-The Rare-Earth Elements, Natl. Bur. Stand. Ref. Data Ser., Natl. Bur. Stand. (U.S.) Circ. No. 60 (U.S. GPO, Washington, D.C., 1978).

${ }^{18}$ F. Gerken, J. Barth, and C. Kunz, Phys. Rev. Lett. 14, 993 (1981).

${ }^{19}$ E. Schmidt, H. Schröder, B. Sonntag, H. Voss, and H. E. Wetzel, J. Phys. B 18, 79 (1985).

${ }^{20}$ S. T. Lee, S. Süzer, E. Matthias, R. A. Rosenberg, and D. A. Shirley, J. Chem. Phys. 66, 2496 (1977).

${ }^{21}$ F. Gerken, J. Phys. F 13, 703 (1983).

${ }^{22}$ Th. Prescher, M. Richter, E. Schmidt, B. Sonntag, and H. E. Wetzel, J. Phys. B 19, 1645 (1985).

${ }^{23} \mathrm{~A}$. Yagishita et al. (unpublished).

${ }^{24}$ Ch. Dzionk, W. Fiedler, M. v. Lucke, and P. Zimmermann, Phys. Rev. Lett. 62, 878 (1989).

${ }^{25}$ M. Meyer, T. Prescher, E. v. Raven, M. Richter, E. Schmidt, B. Sonntag, and H.-E. Wetzel, in Giant Resonances in Atoms, Molecules, and Solids, edited by J. P. Connerade, J. M. Esteva, and R. C. Karnatak (Plenum, New York, 1987), p. 251.

${ }^{26} \mathrm{U}$. Becker, in Giant Resonances in Atoms, Molecules, and Solids, edited by J. P. Connerade, J. M. Esteva, and R. C. 
Karnatak (Plenum, New York, 1987), p. 473.

${ }^{27}$ F. Combet Farnoux, Phys. Rev. A 25, 287 (1982).

${ }^{28}$ U. Fano, Phys. Rev. 124, 1866 (1961).

${ }^{29}$ U. Fano and J. W. Cooper, Phys. Rev. 137, A1364 (1965).

${ }^{30}$ B. W. Shore, Phys. Rev. 171, 43 (1968).

${ }^{31}$ F. H. Mies, Phys. Rev. 175, 164 (1968).

${ }^{32}$ P. C. Kemeny, J. A. R. Samson, and A. F. Starace, J. Phys. B 10, L201 (1977).

${ }^{33}$ L. C. Davis and L. A. Feldkamp, Phys. Rev. B 23, 6239 (1981).

${ }^{34}$ H. G. Kerkhoff (unpublished).

${ }^{35}$ R. D. Cowan, J. Opt. Soc. Am. 58, 808 (1968); The Theory of Atomic Structure and Spectra (University of California Press, Berkeley, London, 1981).

${ }^{36}$ G. K. Wertheim and M. Campagna, Chem. Phys. Lett. 47, 182 (1977).

${ }^{37}$ G. K. Wertheim and G. Crecelites, Phys. Rev. Lett. 40, 813
(1978).

${ }^{38}$ J. W. Allen, L. I. Johanson, R. S. Bauer, I. Lindau, and S. B. M. Hagström, Phys. Rev. Lett. 41, 1499 (1978).

${ }^{39}$ F. Gerken, Dissertation Universität Hamburg, Hamburg, Germany [also available as Internal Report DESYF41-83/03, Hamburg (1983)].

${ }^{40}$ U. Becker, H. G. Kerkhoff, D. W. Lindle, P. H. Kobrin, T. A. Ferrett, P. A. Heimann, C. M. Truesdale, and D. A. Shirley, Phys. Rev. A 34, 2858 (1986).

${ }^{41}$ U. Becker (private communication).

${ }^{42} \mathrm{~F}$. Gerken, J. Barth, and C. Kunz, in X-ray and Atomic InnerShell Physics (University of Oregon, Eugene, Oregon), Proceedings of the International Conference on X-Ray and Atomic Inner-Shell Physics, AIP Conf. Proc. No. 94, edited by B. Crasemann (AIP, New York, 1982), p. 602.

${ }^{43}$ A. Zangwill and P. Soven, Phys. Rev. Lett. 45, 204 (1980). 